\title{
Die Zeichen der Zeit erkennen
}

Hedi Meierhans

Korrespondenz:

Dr. med. Hedi Meierhans

Psychiatrie und Psychotherapie

Schulhausstrasse 5

CH-8722 Kaltbrunn

hedi.meierhans@hin.ch
In der Schweizerischen Ärztezeitung Nr. 47/2002 habe ich einen Artikel unter dem Titel «Wo ist das Feuer des Prometheus geblieben?» veröffentlicht. Es ist mir darum gegangen, anhand von Beispielen aufzuzeigen, dass wir uns von einer veralteten Lebensphilosophie in eine Sackgasse haben treiben lassen und dass die Wurzeln des Problems in unserem Denken, in unseren Definitionen und in unserer Auffassung von Geist und Materie liegen.

Dieser Artikel wäre eine gute Voraussetzung für das Verständnis des folgenden. Die Zeichen der Zeit sehen wir vor allem in den Veränderungen in, um und auf der Erde und am Engelboom. Ich möchte im folgenden nur über den Engelboom reden.

\section{Der Engelboom}

Die Engel sind plötzlich wieder salonfähig geworden, man sieht sie in öffentlichen Parks und in Bahnhöfen. Die herausragendsten sind die von Niki de Saint Phalle. Engelbücher zieren ganze Regale in Buchhandlungen. Engel lächeln von Schirmen herunter oder von Taschen und Auslagen. Ein holländischer Arzt namens Moolenburgh hat ein Buch über Engelbegegnungen geschrieben, nachdem er seine Patienten beiläufig danach befragt hat.

Das alles ist kein Zufall. Künstler sind meist die ersten, die wahrnehmen, was in der Luft liegt oder fliegt. Man spricht ja vom Wassermannzeitalter auch als dem Zeitalter der Engel. Jedoch, was man nicht sehen kann, ist immer noch für viele nicht existent. Und was man nicht sehen kann, kann man auch kaum beschreiben. In den Kirchen werden Engel häufig als menschenähnliche Wesen mit Flügeln dargestellt.

Vor vielen Jahren, des Nachts, sah ich ein Wesen auf mich zukommen, das aussah wie eine grosse weisse Kerze. Die «Flamme» war zwiebelförmig, mit verschiedenen transparenten Schichten, und gegen unten löste sich die «Kerze» allmählich auf. Ich war sehr erstaunt und sah wie gebannt auf das Wesen. Es schwebte auf mich zu und ging durch mich hindurch. Ich hatte keine Angst, es war eher angenehm und beruhigend. Dann war der Spuk vorbei. Ich vergass es wieder. Als ich viele Jahre später in einem Engelbuch von Flower Newhouse die Beschreibung der verschiedenen Engel las, fand ich mein «Wesen» von damals wieder, es hiess Nachtengel und hat eine Schutzfunktion. Mir wäre vorher nie im Traum in den Sinn gekommen, dass es sich da um einen Engel handeln könnte. Wieder einige Jahre später kam ich zufällig zu einem Engelseminar, das von einem Armenier abgehalten wurde. Unter anderem erwähnte der Armenier, dass es 5 verschiedene Arten von Schutzengelbegegnungen gebe:

1. der Engel als Stimme, die aus dem Herzen kommt;

2. als sogenannter «Führungstraum»;

3. als Hund oder Mensch, der plötzlich in Gefahrensituationen anwesend ist, um dann ebenso plötzlich wieder zu verschwinden;

4. als spürbare positive Energie;

5. der Engel als Wesen, die «Flügel» sind Energieströmungen.

Ich bemerkte zu meinem Erstaunen, dass ich fast alles schon erlebt hatte, ohne dabei je einen Gedanken an Engel verloren zu haben. Neben der «Kerze» war das eindrücklichste Erlebnis die Stimme aus dem Herzen. Das kann man nicht beschreiben, nur erleben. Eine Melodie aus der Deutschen Messe von Schubert entsprang meinem Herzen, die ich seit der Kindheit nicht mehr gehört hatte. (Man sagt den Engeln auch die Singenden.) Ich suchte nachher den genauen Text und erkannte die Botschaft. Eine andere Erfahrung fand an einem Wochenenddienst in der Stadt Zürich statt. Ich kam nach Mitternacht in meine Praxis zurück und war fix und fertig. Ich legte mich auf die Couch und sagte für mich: «Jetzt müssen mir alle guten Geister helfen, ich mag nicht mehr.» Augenblicklich wurde ich von einer feinen, mütterlichen Energie wie zugedeckt. Ich fühlte mich völlig geborgen und ruhig.

An den Engeln kann ich mit dem besten Willen nicht mehr zweifeln. Inzwischen weiss ich auch, dass die in den Religionen beschriebenen Engel wirklich existieren und für uns und unsere Erde lebenswichtige Funktionen erfüllen. Sie warten nur darauf, dass wir wieder mit ihnen in Kontakt treten, sie möchten uns gerne helfen, wenn wir wollen, sie lassen uns den freien Willen.

Ein christlicher Eingeweihter aus Bulgarien sprach einst von der Nahrungskette: Die Pflanze nährt sich vom Mineral, das Tier von der Pflanze und kleineren Tieren, der Mensch ist ein Omnivor, und wer nährt sich von uns? Unsere Gedanken und Gefühle sind Nahrung für die Engel! Ohne 
die Engel wären wir Menschen schon lange im eigenen «Müll» erstickt. Die Engel wandeln dauernd unsere negativen Energien um (unter anderem). Sie nähren sich von positiven Energien, aber natürlich nicht nur von uns, sonst wären sie schon lange verhungert. Einen Teil unserer Negativität müssen wir aber selbst ausbaden. Das sehen wir auch, wenn wir die Augen offen haben.

\section{Spiegelneuronen}

Definition: Nervenzellen, die im Gehirn beim Beobachten einer Tätigkeit (auch eines Traumas) die gleichen Potentiale auslösen, wie wenn eine Tätigkeit selbst durchgeführt würde. Die Hauptzentrale der Spiegelneuronen sitzt im präfrontalen Cortex.

Gedanken und Gefühle sind also nicht nichts, es sind starke Energien, die dauernd von uns ausstrahlen. Wir haben noch lange nicht alle Energien entdeckt, wir werden sie erst entdecken können, wenn wir unsere eigenen feineren Instrumente in Betrieb nehmen können.

Sind die Gedanken und Gefühle positiv, fühlen wir uns wohl und fit, sind sie negativ, kennen wir das auch. Kein Arzt wird leugnen können, dass die Psyche sich auf unseren Körper auswirkt. Zum grössten Teil sind es unsere Gefühle und Gedanken, die für unseren Gesundheitszustand verantwortlich sind. Wenn wir uns schlecht fühlen, sind wir meist in einem falschen Muster, zeigen sich unsere «Wunden». Wenn wir unser Denken nur einmal einen Tag lang beobachten, sehen wir, wo wir mit der Prävention ansetzen müssen. Die falschen Muster werden uns von Generation zu Generation übertragen in unserer Kindheit. Die Spiegelneuronen in der linken Gehirnhälfte des limbischen Systems sind bei der Geburt nicht mit Informationen besetzt. Der göttliche Geist befruchtet nach der Geburt die rechte Hirnhälfte, bis die Fontanelle $\mathrm{zu}$ ist (St.-Germain). Die rechte Hirnhälfte wird zu $100 \%$ genutzt und enthält den Bauplan des Lebens. Wenn das Kind aus seinem unbewussten göttlichen Gefühl heraus anfängt, seine Umwelt wahrzunehmen und zu benennen, speichert es seine Schlüsse in der linken Hirnhälfte und besetzt die Spiegelneuronen mit mentalen Gedankenstrukturen. Der Gyrus cinguli bewertet jedes empfangene Bild des Kindes. Die erste Schlussfolgerung, die der Gyrus cinguli über seine gesammelte Erfahrung gebiert, steht mit dem Wertgefühl des Kindes in direkter Verbindung. Der Gyrus cinguli der rechten Hirnhälfte des Kindes übermittelt seiner linken Hirnhälfte über das Corpus callosum jede geborene Schlussfolge- rung. Wenn ein Kind zur Schlussfolgerung der Wertlosigkeit kommt, löst das im Gehirn einen Alarm aus, der zur Ausschüttung von Cortisol führt.

Die Spiegelneuronen der rechten Hirnhälfte kopieren hauptsächlich die Emotionen der Mutter. Die Spiegelneuronen der linken Hirnhälfte kopieren hauptsächlich die Gedankenstrukturen des Vaters. Bis 21 hat es etwa 95\% der Spiegelneuronen mit Informationen besetzt. Wenn ein Kind bis 5 Jahre nicht mindestens $50 \%$ seiner Spiegelneuronen mit Informationen besetzt hat, ist es nicht überlebensfähig. Unsere derzeitige Nutzungskapazität ist aber höchstens $12-15 \%$ in der linken Hirnhälfte. Wenn das Kind durch die falschen Muster der Umwelt oder Dogmen falsche Schlüsse zieht, z. B. in seinem richtigen göttlichen Gefühl nicht bestätigt wird, gebiert es in seine linke Hirnhälfte falsche Gedankenstrukturen, die sich dann als falsche Muster bzw. psychische Wunden äussern. Die falschen Schlüsse aus der Umwelt erhöhen die mentale Intelligenz nicht. Wenn die Forschung nicht der Wahrheit und dem Fortschritt dient, bringt sie keinen wirklichen Fortschritt. Jesus Christus hatte eine 100prozentige Nutzungskapazität der linken Hirnhälfte, und wir sollten ihm nachfolgen ... Er hat Gottes Geist zu 100\% gespiegelt! Das heisst, der göttliche Geist ist in die Materie geboren worden, durch seine, durch unsere Selbstbewusstwerdung, durch das Übersetzen des göttlichen Plans in unsere mentalen Strukturen in der linken Hirnhälfte; diese Geburt ist bei uns noch nicht abgeschlossen. Wir alle sind göttliche Wesen im Status nascendi. Ausserdem sitzt der göttliche Funke als Vater-Sohn-Geist-Prinzip in jedem Menschenherz. Darum wurde Jesus auch oft mit einem flammenden Herzen dargestellt. Das unterscheidet uns vom Tier. (Die dreifältige Flamme sitzt natürlich nicht im Herzmuskel, oder glaubt jemand allen Ernstes, dass die Liebe oder das Glücksgefühl aus dem Herzmuskel kommt? Die Medizin wird nicht darum herumkommen, ihr Konzept durch die «Anatomie und Physiologie» der Seele zu erweitern.)

\section{Die Illusion der Trennung}

Ein Englischlehrer erzählte mir einmal, dass er Shakespeare für den grössten Dichter halte, weil er das Hauptdrama immer in Nebendramen spiegle.

Wir schaffen uns unsere Dramen selbst. Was der andere, mit dem wir in Konflikt stehen, uns spiegelt, hat mit uns selbst zu tun. Aber auch das Positive, das er uns spiegelt, hat mit uns zu tun. Wir leben in einem einheitlichen göttlichen Psyche-Geist-Feld, das schon der Physiker Jules 
Muheim postuliert hat. Wenn die Affen auf einer Insel anfangen, die Kartoffeln vor dem Essen zu waschen, und bald darauf andere auf einer entfernten Insel auch, wird das mit dem etwas zu tun haben. Wahrscheinlich wird dies die weitere Erforschung in der Neurobiologie, auch der Spiegelneuronen, bestätigen. Ich vermute, dass das Prinzip der Resonanz und das Prinzip der Spiegelung die Schlüsselprinzipien in der Forschung darstellen. Diese Prinzipien sind überall anzutreffen: in der Musik, in der Physik, Mathematik, Neurobiologie, in den zwischenmenschlichen Beziehungen usw.

Eindrücklich ist das Gesetz der Resonanz: Wenn uns etwas sehr beschäftigt, sei es positiv oder negativ, sehen wir es überall, vorher haben wir etwas, an dem wir seit Jahren vorbeigegangen sind, gar nicht bemerkt. Wir sind immer dort, wo unser Bewusstsein fokussiert ist. Es ist deshalb auch so wichtig, auf was wir fokussiert sind. Wir ziehen das an, was wir, auch unbewusst, ausstrahlen. Das menschliche Leben ist ein Bewusstwerdungsprozess. Diejenigen, die in der Unbewusstheit verharren wollen, blockieren ihre Entwicklung. Jedoch in unserer Essenz sind wir nie aus der göttlichen Einheit gefallen. Nur unser Körper ist so dicht geworden, dass er für direkte Lichtnahrung nicht mehr zugänglich ist. Meist projizieren wir unsere Wunden lange nach aussen, bis wir merken, was abläuft. Die häufigsten Wunden in der Partnerschaft sind die des Nicht-anerkannt-Werdens und des Nicht-akzeptiert-Werdens. Diejenigen Menschen, die z.B. auch in der Therapie mitarbeiten, ihre Wunden bewusst machen und die falschen Muster umpolen, leisten eine wichtige Bewusstseinsarbeit und nehmen ihren negativen Anteil aus dem kollektiven Unterbewusstsein heraus. Wir sind bis zu einem hohen Grad die Schöpfer unserer eigenen Realität, so unbequem das vielleicht klingen mag. Bevor die Kriege aufhören, müssen wir Bilderstürmer in der eigenen Seele werden.

Das «richtige Denken und Fühlen» wurde schon in Buddhas achtgliedrigem Pfad gelehrt. Das Vergeben spielt eine grosse Rolle. Wenn wir es nicht tun, tragen wir die negative Energie dauernd in uns herum. Mit der Zeit schädigt das unsere Organe. Die tiefsten Wunden entstehen durch den sexuellen Missbrauch. Da kann z. B. ein daraus folgendes unbewusstes Nein zur Sexualität oft lange ein Hindernis sein, das erst erkannt wird, wenn der Mensch bereit ist, den Spiegel von aussen zu betrachten, um zu erkennen, dass das Nein von ihm selbst kommt. Das Aussen spiegelt, wie wir selbst mit uns umgehen. Es braucht aber viel Geduld, um die Ablehnung und Wut in Akzeptanz und Vertrauen umzuwan- deln. Das geht manchmal bis zur Resignation. Jede Erfahrung, sei sie noch so schlimm, erweitert jedoch immer das Bewusstsein, wenn wir sie überstehen und annehmen. Der Mensch lotet mit seiner Seele die irdische Welt tief aus, ein Baum, der tiefe Wurzeln macht, hat eine grosse Krone. Durch die eigene Erfahrung und die daraus folgende Erkenntnis bringt der Mensch Licht in die Dunkelheit.

Die Dramen auf der Welt begannen schon bei Adam und Eva. Sie fingen an mit der Illusion von Getrenntheit und Bewertung. Erst wenn wir begriffen haben, dass alles aus der gleichen Quelle entspringt, unser Geist ein Teil des göttlichen Geistes ist, das ganze sichtbare und unsichtbare Universum aus Atomen besteht, die wiederum wellenförmige Energiezustände darstellen können, fängt das bewusste Leben an und ist es möglich, die richtigen Prioritäten zu setzen und uns nach den göttlichen Gesetzen zu richten. Wobei die Sichtbarkeit von der Geschwindigkeit abhängt, mit denen die Elektronen den Atomkern umkreisen oder mit der ein Atom schwingt. Das ganze Universum mit seinen wunderschönen Galaxien ist der Körper des kosmischen Christus, darum sind wir in ihm und er in uns.

Wir dürfen alle Fehler machen, wir werden von «oben» niemals abgewertet, wichtig ist nur, dass wir bereit sind zu lernen, sonst lehrt uns das Leben, bis wir verstehen. Aus den Bewertungen entsteht der Minderwert, der so viel Übel produziert. Das Abwerten der andern geschieht immer aus der Wunde des eigenen «Minderwerts», dabei sind wir alle göttliche Wesen. Der göttliche Geist ist unteilbar und vollkommen, alle Söhne und Töchter Gottes sind in Wahrheit Licht und Liebe. Selbst die Theologen sagen, es gebe weder Mann noch Frau noch Kind, wir seien alle eins in Christo. Weil wir das aber leider noch nicht spüren, fühlen wir uns getrennt. Was ich dem «andern» scheinbar antue, habe ich mir aber selbst scheinbar angetan. Die materielle Welt ist eine Scheinwelt, weil sie aus projizierten «geronnenen Gedanken» besteht, die uns scheinbar von Gott trennt. Wenn wir den andern in seiner Gottheit nicht anerkennen, verleugnen wir Gott und uns selbst. Aus unseren scheinbaren Wunden heraus, die schon seit Generationen auf uns übertragen wurden und nicht einmal unsere eigenen sind, werten wir ab. Das mit dem Verstand erkennen ist aber erst der erste Schritt zur Heilung, die Heilung erfolgt erst, wenn das Herz begriffen und verziehen hat.

Unsere Vergehen sind scheinbare Vergehen, weil die materielle Welt eine Illusion ist, an die wir leider glauben. Wir brauchten gar keine 
Schuldgefühle zu haben. Wenn uns jemand scheinbar etwas antut, spiegelt er den Irrtum unseres Geistes, der nicht weiss, was er tut oder einmal getan hat, und vergessen hat. Darum liegt die Heilung von uns selbst im Verzeihen, denn wenn wir dem «andern» verzeihen, verzeihen wir uns selbst und machen uns frei. Das heisst, wir müssen im Geist dem Angreifer sagen, wer er ist, ein Kind Gottes, und dass wir ihm verzeihen, dass er das vergessen hat, und wir auch. Das Ego ist ein Kunstprodukt aus den Erfahrungen der Scheinwelt, nur das verzerrte Spiegelbild unseres göttlichen Ichs. Es ist daher verletzlich. Das wahre göttliche «Ich bin» ist unverletzbar (das habe ich sogar einmal in einem bestimmten Moment erlebt). Darum sollten wir uns auch nicht verteidigen, denn damit anerkennen wir die Scheinwelt und leugnen Gott. Darum hat sich auch Jesus nicht verteidigt, sondern gesagt: «Vater, vergib ihnen, denn sie wissen nicht, was sie tun.» Jesus hat die ganze Zeit seine Konzentration auf seine göttliche Ich-bin-Gegenwart gerichtet, der materiellen Welt die Macht genommen über sich, nur darum konnte er auferstehen. Aber: Wir sind hier auf das göttliche Paradoxon gestossen: Der absolute Gesichtspunkt zeigt die eine Seite der Medaille - die andere Seite ist die Relation. Absolute Wahrheit wurde definiert als «Dinge, wie sie der Geist Gottes erkennt», dagegen relative Wahrheit als «Dinge, wie sie die höchste Vernunft des Menschen erkennt». Solange wir auf dem materiellen Plan leben, ist die Materie für unsere Sinne eine Tatsache. Wir müssen lernen, die Materie durch die Anwendung höherer Kräfte und Gesetze zu beherrschen, aber nicht die Existenz der Materie im relativen Aspekt verneinen. Unsere Vergehen sind nur scheinbare Vergehen vom absoluten Gesichtspunkt aus gesehen.

Dies gilt vor allem für das Individuum, das aus dem Alptraum der Materie erwachen will, in Übergangszeiten ist es aber nicht ratsam, z. B. die ganze Verteidigungsarmee abzuschaffen, weil sonst der Alptraum sich unnötig verlängern könnte. Noch zu viele schlafen fest in der Materie und spielen ihr Spiel, anerkennen nur die relative Wahrheit. Auch die Schulmedizin werden wir noch ein Weilchen brauchen. Der Körper sagt uns zwar, was wir falsch machen, aber wir können oder wollen es nicht immer sehen. Schon Tilmann Moser kam in seinem Buch «Lehrjahre auf der Couch» zum Schluss, dass es die Liebe war, die heilte. Ja, die wahre Heilung liegt in der Anwendung der bedingungslosen Liebe, das weiss ich seit 30 Jahren, aber begriffen habe ich es erst jetzt. Wir können sie in unserem Herzen aktivieren.
Aber auch wenn wir dies alles wissen, können wir es zur Zeit noch nicht ganz umsetzen, wir werden immer wieder in die Unbewusstheit, auf die Bühne der Dualität gezogen, weil die Unbewusstheit momentan noch sehr gross ist. Erst nach dem «grossen Weihnachten» oder, wie die Mayas prophezeiten, nach der Wiederkunft der Götter, die das gleiche Ereignis anspricht wie die Wiederkunft Christi, wird das leichter fallen, dann macht auch unsere linke Hirnhälfte einen «Quantensprung». Aber immerhin kann uns das Wissen um gewisse Dinge die Richtung zeigen, und Übung macht den Meister.

Warum wollten wir die Illusion der Getrenntheit erfahren? Wir wüssten nicht, was Vergessen ist, was Verlassenheit ist, was Schmerz ist, aber auch nicht, was irdische Liebe ist, was Schönheit ist, was Sieg ist usw. Bevor es eine Biologie gab, existierte nur Stern und Stein. Das eine war zu flackernd, das andere zu fest. Das heisst, ohne Biologie wären wir nie beständige und doch flexible, bewusste «Ich bin» geworden. Diese Entwicklung ist nur in der Biologie auf einem Planeten möglich. Die ich- und gottbewusste Seele kehrt als ichbewusster göttlicher Funke in die geistige Welt zurück, wo sie neben der Einheit mit Gott (wenn sie erwacht ist aus dem Traum der Materie) auch die eigene Individualität spürt. Man könnte sich vorstellen, jeder Mensch ist eine Melodie Gottes, durch die Erdenleben hat der Mensch eine Symphonie daraus gemacht. (Himmel und Erde werden vergehn, aber Frau Musica bleibet bestehn.) Aber warum wir uns wirklich von Gott trennen wollten, ist für mich damit noch nicht ganz klar, warum haben wir uns das angetan? Hat unsere Schöpfermacht uns soweit getrieben?

Wir sind schon sehr lange auf dem Weg und hätten jetzt die Möglichkeit, den Heimweg anzutreten, aber ganz allein würden wir es nicht schaffen. Wir trafen vor sehr langer Zeit eine Abmachung: Wenn wir einen bestimmten Bewusstheitsgrad erreicht haben würden, würden uns die Engel aufwecken. Das heisst, wir wissen wieder, wer wir sind, dass die Trennung von Gott eine Illusion ist. Die Engel sind am Werk! Das ist der erste Schritt, der zweite folgt in der Wiederkunft, das heisst in der Geburt des Christusbewusstseins in uns. Angelus Silesius sagte: «Und wäre Christus tausendmal in Bethlehem geboren, und nicht in uns, wir wären ewiglich verloren.»

Es gibt gute Hilfsmittel, um unser Sein zu harmonisieren, um uns vorzubereiten, z. B. das Forschen nach Erkenntnis, nach Selbsterkenntnis, d.h. die Suche nach der Wahrheit. Die Schönheit der Natur oder die Musik, sie ist ja 
auch die geistigste der Künste, und die grossen Komponisten wie Mozart, Beethoven, Brahms usw., die sich beim Komponieren mit Gott in Verbindung wähnten, waren mit Engeln in Verbindung, denn Gott hat seine Diener überall, wir können Ihn momentan vor allem über seine Hierarchien erfahren. Und unsere nächsten Brücken zu Ihm sind unsere Schutzengel. Interessanterweise geben die Wiener Walzer von Johann Strauß, insbesondere der Kaiserwalzer, der Seele Flügel, sie haben eine Resonanz zu der hohen Wesenheit, die für die nächsten 2000 Jahre einen grossen Einfluss auf die Evolution haben wird wie vorher Jesus Christus (Die Welt und «der Himmel» sind nicht stillgestanden!). Das Lied «Abends wenn ich schlafen geh» aus der Oper «Hänsel und Gretel» hat eine Resonanz zu den Schutzengeln ... und Händel erzählte, dass sich nach der Vollendung seines «Messias» «der Himmel geöffnet hat ...»

Seit mindestens 40 Jahren ist mir bewusst, wie wichtig die Kontrolle der Gefühle und Gedanken ist, aber die ganze Tragweite habe ich erst jetzt erkannt, weil ich jetzt weiss, dass unsere negativen Emotionen uns bis zu den Elektronen verschmutzen und verdichten, und weil wir das werden, was wir denken. Der Physiker Jules Muheim hat ja schon gesagt, dass kein Atom jemals vergisst, was ihm alles zugestossen ist. Darum ist es auch so wichtig, was für Prioritäten wir setzen, auch in der Verteilung der Forschungsgelder und im Gesundheitswesen. Psychotherapie ist kein Luxus.

Gibt es denn etwas Wichtigeres als die Gesundheit von Seele und Körper und das Erwachen aus dem Alptraum in der Materie? (Auch wenn der Planet Erde wunderschön ist und seine Nahrung herrlich.) Es ist für jeden Menschen wichtig, seinen Weg zu gehen, es führen bekanntlich immer verschiedene Wege nach Rom, Spiritualität ist nur einer davon. Es gibt aber geistige Gesetze, die für alle gelten.

\section{Ausblick}

Wir leben in einer Zeit der Transformation, der wir am besten mit Mut, Wachsamkeit und Gelassenheit entgegengehen. Konflikte spielen sich immer auf der Ebene von Bildern, die wir voneinander haben, ab. Nicht auf der Ebene unserer wahren Essenz (die Licht [Wahrheit] und Liebe ist). Aufstände zeigen die Wunden einer Volksgruppe, die nach aussen projiziert werden. Die Wunden des männlichen Prinzips sind: Zorn,
Hass, Konkurrenz, Kampf, Aggression, Bewertung. Die des weiblichen: Schuldgefühle, Selbstzweifel. (Diese «Wunden» entstanden aus der Illusion der Trennung.) Sie schreien nach Heilung, die aber nicht mit Gewalt geschehen kann, sondern durch Mitgefühl und Verzeihung. Sie zeigen uns, was wir falsch machen. Wir müssen erkennen, dass, wenn wir in einen Kampf verwickelt sind, wir immer eine Resonanz in unserem Inneren dazu haben, eine scheinbare Wunde, die von uns erlöst werden will durch Mitgefühl zu uns selbst und anderen. Die Erde ist unser Übungsplanet, der uns nicht gehört. Er ist unsere Amme, der wir gut schauen dürften.

Wir können niemanden ändern ausser uns selbst, weil der andere uns spiegelt. Wir möchten aber häufig, dass der andere sich ändert. Wir können auch keine Erfahrungen machen für andere, wir müssen sie selbst machen. Unser Bewusstsein erweitert sich mit Erfahrung, mit Erproben von Wissen.

Wir stehen vor dem grössten Abenteuer, das die Menschheit je erlebt hat, wir stehen davor, unser wahres Erbe anzutreten. Die Spreu wird vom Weizen getrennt, weil sie sonst noch sehr lange die Menschen, die guten Willens sind, hindern würden. Das hat u.a. ausser Jesus auch Rudolf Steiner, der einer der grössten spirituellen Lehrer war, schon Anfang des letzten Jahrhunderts gesagt. Die «Spreu» wird eine ziemlich lange «Ehrenrunde» an einem andern Ort machen müssen, bis sie zum Weizen wird. Jesus Christus sagte nicht umsonst, wir seien auch Götter und könnten einmal die Werke tun, die er tut und noch mehr. Aber wir müssen vorbereitet sein und den Staub der Jahrtausende, der auf unserem Bewusstsein liegt, abschütteln. Auch das Gleichnis mit den klugen und törichten Jungfrauen weist darauf hin, dass wir «Öl in unseren Lampen» haben müssen, wenn der «Bräutigam» kommt, der ein Symbol für das göttliche Bewusstsein ist, das in der Biologie erwachen will. Die Türe, die für die törichten Jungfrauen verschlossen ist, ist nicht aus Bosheit oder Kleinlichkeit geschlossen, sondern weil dann einfach keine Zeit mehr da ist für Erklärungen und Unterweisungen. Wer das neue Bewusstsein, das heisst auch stärkeres Licht, fassen kann, der fasst es. Unser Körper mit seinem Nervensystem ist zur Zeit wie eine Glühbirne, die noch nicht am Strom angeschlossen ist. Die «Re-ligio» der $\mathrm{Zu}-$ kunft ist überkonfessionell und steht auf dem Boden des Wissens und der Erfahrung. 